Z. klin. Chem. u. klin. Biochem.

9. Jg., S. 238-241, Mai 1971

\title{
Eine Methode zur Bestimmung des freien Ätiocholanolons im Plasma
}

\author{
Von V. GraeF \\ Aus dem Biochemiscben Institut der Universität Gießen (Direktor: Prof. Dr. Hj. Staudinger)
}

(Eingegangen am 4. Dezember 1970)

Eine Methode zur Bestimmung des freien Ätiocholanolons im Plasma wird beschrieben. Durch Dünnschichtchromatographie auf Aluminiumoxid wird das Ätiocholanolon aus dem Plasmaextrakt isoliert. Nach Acetylieren der 3-Hydroxygruppe und Reaktion der 17Oxogruppe mit Dansylhydrazin wird das Acetyl-ätiocholanolon-dansylhydrazon durch eine 2. Dünnschichtchromatographie auf Kieselgel abgetrennt. Die Fluoreszenz dieses Derivates wird gemessen. Mit dieser Methode können noch 0,006 $\mu$ g Ätiocholanolon bestimmt werden.

\section{A method for the determination of free aetiocholanolone in plasma}

A method for the determination of free aetiocholanolone in plasma is described. The aetiocholanolone is isolated.by thin layer chromatography on aluminium oxide. After acetylation of the 3-hydroxy-group and reaction of the 17-oxo-group with dansylhydrazine the acetylaetiocholanolone-dansylhydrazone is separated by thin layer chromatography on silica gel. The fluorescence of this derivative is measured. As little as $0.006 \mu \mathrm{g}$ aetiocholanolone can be determined by the above method.

Die Bestimmung des freien, unkonjugierten Ätiocholanolons im Plasma wird zur Diagnose des sog. Ätiocholanolonfiebers benutzt. Die pyrogene Wirkung des Ätiocholanolons wurde 1957 von Segaloff (1) und von Kappas (2) erkannt. Normalerweise kommt das freie Ätiocholanolon nur in sehr geringen Konzentrationen im Plasma vor. Die meisten Autoren fanden Wertè unter $1 \mu \mathrm{g} / 100 \mathrm{ml}$. Während der periodisch auftretenden Fieberanfälle steigt der Gehalt des Plasmas an freiem Ätiocholanolon auf ein Mehrfaches der normalen Konzentration an. Das freie Ätiocholanolon wurde bisher mit einer Doppelisotopenverdünnungsmethode (3) und einer gaschromatographischen $\mathrm{Me}$ thode (4) bestimmt. Wegen der geringen Konzentration läßt sich die Zimmermannsche Farbreaktion auf 17Oxosteroide nicht verwenden; auch mit der MikroZimmermanN-Reaktion lagen die Werte unter der Nachweisgrenze der Methode (5). Durch Überführung des Ätiocholanolons in das gelbgefärbte 2,4-Dinitrophenylhydrazon wurde die photometrische Bestimmung kleinerer Mengen dieses Steroides möglich $(6,7)$.

Wie wir kürzlich berichteten (8), kann man Oxosteroide mit Dansylhydrazin in die stark fluoreszierenden Dansylhydrazone überführen. Diese Reaktion wurde nun zur Bestimmung des freien Ätiocholanolons im Plasma angewendet. Bei Verwendung von Mikroküvetten liegt die untere Nachweisgrenze bei $0,006 \mu \mathrm{g}$ Ätiocholanolon in einer Probe.

\section{Methodik}

\section{Reagenzien}

Diäthyläther: DAB 6 (E. Merck, Darmstadt) wird stets frisch über Natriumdraht destilliert.

Methylenchlorid p. a. (E. Merck, Darmstadt), Essigsäureäthylester p. a. (E. Merck, Darmstadt) und Benzol p. a. (E. Merck, Darmstadt) werden mit je $1 \mathrm{~g} \mathrm{2,4-Dinitrophenylhydrazin} \mathrm{und} 1 \mathrm{~g}$ Trichloressigsäure/l destilliert. Um zu verhindern, daß Dinitro- phenylhydrazin mitgerissen wird, setzt man eine kleine mit Raschig-Ringen gefüllte Kolonne auf den Destillationskolben auf. Chloroform: p. a. (E. Merck, Darmstadt).

n-Hexan: rein (E. Merck, Darmstadt).

Methanol: p. a. (E. Merck, Darmstadt) wird über Natriumborhydrid $(2 \mathrm{~g} / l)$ destilliert und dann an einer Füllkörperkolonne redestilliert.

Aluminiumoxid: $\mathrm{GF}_{254}$ für Dünnschichtchromatographie (E. Merck, Darmstadt).

Kieselgel H: für Dünnschichtchromatographie (E. Merck, Darmstadt).

Dünnschichtplatten: Glasplatten $(20 \times 20 \mathrm{~cm})$ werden mit einer $0,25 \mathrm{~mm}$ dicken Schicht Aluminiumoxid $\mathrm{GF}_{254}$ bzw. Kieselgel $\mathrm{H}$ versehen, 3 Stdn. im Trockenschrank bei $110^{\circ}$ erhitzt und in einem Exsikkator ohne Trockenmittel aufbewahrt.

Ätiocholanolon (Vister, Casatenovo Brianza (Como)).

Progesteron (E. Merck, Darmstadt).

$17 \alpha$-Hydroxyprogesteron (E. Merck, Darmstadt).

1,1,2,2-Tetrachloräthan (E. Merck, Darmstadt).

Dansylhydrazin (1-Dimethylamino-naphthalin-5-sulfonylhydrazin): Die Darstellung erfolgt nach der in einer frühereñ Arbeit angegebenen Vorschrift (8).

Progesteron-bis-(dansylhydrazon)-Lösung: Man verdampft in einem spitzen Zentrifugenröhrchen $2 \mathrm{ml}$ Progesteron-Lösung ( $1 \mathrm{mg} / 100 \mathrm{~m} /$ Chloroform), versetzt mit $1 \mathrm{ml}$ DansylhydrazinLösung $(2,4 \mathrm{mg} / 25 \mathrm{ml}$ Benzol) und $1 \mathrm{ml}$ TrichloressigsäureLösung ( $30 \mathrm{mg} / 100 \mathrm{ml}$ Benzol), dampft die Lösung nach $30 \mathrm{Min}$. am Rotationsverdampfer ein und trägt den Rückstand mit Essigester in Form mehrerer, dicht nebeneinander liegender Punkte auf eine Kieselgel-H-Platte auf, die im System Benzol/Essigester $(80: 20 \mathrm{v} / \mathrm{v})$ entwickelt wird. Die unter der UV-Lampe fluoreszierende Zone wird ausgekratzt. Das Kieselgel eluiert man zweimal mit je $2,5 \mathrm{ml}$ Chloroform. Die vereinigten Eluate werden eingedampft, den Rückstand löst man in $20 \mathrm{~m} /$ Benzol.

Alle Glasgeräte werden mit einer 5 proz. Lösung von Lensex (Deutsche Shell-Chemie, Frankfurt/M.) gereinigt und gründlich mit warmem Leitungswasser und dest. Wasser gespült.

\section{Extraktion des Plasmas}

Blut wird unter Zusatz von Heparin $(0,2 \mathrm{ml}=200 \mathrm{E} / 20 \mathrm{ml}$ Blut) abgenommen und sofort zentrifugiert. $10 \mathrm{~m} l$ Plasma werden mit $3 \mathrm{ml} 1 \mathrm{~N} \mathrm{NaOH}$ versetzt und einmal mit $50 \mathrm{ml}$ und einmal mit $30 \mathrm{ml}$ Methylenchlorid/Äther $(1: 4 \mathrm{v} / \mathrm{v})$ extrahiert. Man schüttelt 
die vereinigten Extrakte zweimal mit je $10 \mathrm{ml}$ dest. Wasser aus. Dem 2. Waschwasser setzt man einen Tropfen Eisessig zu. Die Methylenchlorid- $\AA$ ther-Lösung wird über wasserfreiem Natriumsulfat getrocknet und dann am Rotationsverdampfer eingedampft. Den Rückstand versetzt man mit $7 \mathrm{ml}$ Methanol und $3 \mathrm{ml}$ dest. Wasser, überführt die Lösung nach Abkühlen in einen Schütteltrichter und spült mit $5 \mathrm{ml} 70$ proz. wäßr. Methanol nach. Die wäßr.-methanol. Lösung wird mit $5 \mathrm{ml} \mathrm{n}$-Hexan ausgeschüttelt. Nach kurzem Zentrifugieren saugt man die Hexanschicht ab und dampft die wäßr.-methanol. Lösung am Rotationsverdampfer bei $40^{\circ}$ ein. Der Rückstand wird mit $4 \mathrm{ml}$ Åthanol abgedampft und dann mit dreimal je $2 \mathrm{~m} /$ Chloroform in ein spitzes Zentrifugenröhrchen überführt. Diese Lösung dampft man dann ebenfalls ein.

\section{Dïnnscbicbtchromatographie}

Man trägt den Verdampfungsrückstand des Plasmaextraktes mit dreimal $100 \mu l$ Chloroform auf eine Dünnschichtplatte (Aluminiumoxid $\left.\mathrm{GF}_{254}\right) 2 \mathrm{~cm}$ vom unteren Rand entfernt auf. $\mathrm{Da}$ neben trägt man als Bezugssubstanz $3 \mu \mathrm{g} 17 \alpha$-Hydroxyprogesteron in $20 \mu l$ Chloroform auf und entwickelt die Platte drei$\mathrm{mal}$ im System Benzol/Methylenchlorid/Essigester (74:32:16 v/v). Das $17 \alpha$-Hydroxyprogesteron besitzt in diesem System den gleichen $R_{\mathrm{F}}$-Wert wie Atiocholanolon. Es ist im Gegensatz zum Ätiocholanolon bei Betrachtung der Platte unter der UV-Lampe sichtbar. Die Ätiocholanolon enthaltende Zone des Plasmaextraktes wird anhand des Referenzflecks unter der UV-Lampe markiert. Man kratzt das Aluminiumoxid aus und eluiert es in einem Zentrifugenröhrchen mit dreimal je $2,5 \mathrm{ml}$ Chloroform. Nach Zentrifugieren (5 Min. bei 3000 U./Min.) pipettiert man den Uberstand in ein spitzes Zentrifugenröhrchen, in dem es eingedampft wird. Es ist darauf zu achten, daß kein Aluminiumoxid mit überpipettiert wird.

\section{Derivatbildung}

$\mathrm{Zu}$ dem Verdampfungsrückstand des Eluates gibt man $0,4 \mathrm{ml}$ Acetanhydrid und $0,1 \mathrm{~m} l$ Pyridin, mischt durch und läßt verschlossen über Nacht stehen. Ein weiteres Spitzröhrchen, das als Standard dient und $0,1 \mu \mathrm{g}$ Atiocholanolon enthält, wird mit den gleichen Mengen Acetanhydrid und Pyridin versetzt. Am nächsten Morgen pipettiert man in die Röhrchen je $1 \mathrm{ml}$ Methanol und dampft nach $10 \mathrm{Min}$. am Rotationsverdampfer (Olpumpe) ein. In den Röhrchen dampft man anschließend je $1 \mathrm{~m} l$ Progesteronbis-(dansylhydrazon)-Lösung ein und versetzt sie dann mit je $100 \mu l$ Dansylhydrazin-Lösung $(0,48 \mathrm{mg} / 10 \mathrm{ml}$ Benzol $)$ und $100 \mu l$ Trichloressigsäure-Lösung $(1,5 \mathrm{mg} / 10 \mathrm{ml}$ Benzol). In einem als Leerwert dienenden Spitzröhrchen verdampft man ebenfalls $1 \mathrm{~m} l$ Progesteron-bis-(dansylhydrazon)-Lösung sowie je $100 \mu l$ Dansylhydrazin- und Trichloressigsäure-Lösung. Nach 30 Min. werden die Lösungen am Rotationsverdampfer eingedampft.

\section{Dïnnschichtchromatograpbie}

Man trägt den Verdampfungsrückstand in den Spitzröhrchen mit dreimal $100 \mu l$ Essigester punktförmig auf eine Kieselgel-HPlatte auf. Nach zweimaliger Entwicklung der Platten im System Benzol/Essigester $(90: 10 \mathrm{v} / \mathrm{v})$ wird anhand des unter der UVLampe sichtbaren Flecks von Acetyl-ätiocholanolon-dansylhydrazon diese Zone von Hauptwert, Standard und Leerwert ausgekratzt. Das Kieselgel wird mit einer Glasperle und je $2,5 \mathrm{ml}$ Chloroform versetzt und $5 \mathrm{Min}$. geschüttelt. Dann zentrifugiert man $5 \mathrm{Min}$. bei $3000 \mathrm{U}$./Min. und pipettiert das Eluat in Zentrifugenröhrchen, in denen es eingedampft wird. Das Kieselgel wird noch zweimal in der gleichen Weise eluiert, die Eluate dampft man in den gleichen Zentrifugenröhrchen ein.

Man löst den Rückstand in den Zentrifugentöhrchen in $1 \mathrm{ml}$ Tetrachloräthan und mißt die Fluoreszenz in Quarzküvetten MF 4 (1 cm Schichtdicke, $1 \mathrm{ml}$ Fassungsvermögen; Fa. Carl Zeiss, Oberkochen) mit dem Zeiss-Spektrofluorometer. Anregung $362 \mathrm{~nm}$, Fluoreszenz $511 \mathrm{~nm}$; Spaltbreite 1,2/0,5 mm. Zur Berechnung wird die Fluoreszenz des Leerwertes von der des Hauptwertes und des Standards abgezogen.
Die Messung der Fluoreszenz ist auch in einem Photometer „Eppendorf“ mit Fluoreszenzzusatz mög]ich. Hierbei beträgt das Volumen der zu messenden Lösung $2,5 \mathrm{ml}$. Man verwendet das Primärfilter $313+366 \mathrm{~nm}$ und das Sckundärfilter $470-3000 \mathrm{~nm}$.

\section{Ergebnisse}

\section{Sperifität}

Bei der 1. Dünnschichtchromatographie auf Aluminiumoxid $\mathrm{GF}_{254}$ wird Ätiocholanolon von den meisten Oxosteroiden getrennt, jedoch besitzt $17 \alpha$-Hydroxyprogesteron den gleichen $R_{\mathrm{F}}$-Wert (Tab. 1). Um das

Tab. 1

$R_{\text {F-Werte verschiedener Oxosteroide, Dünnschichtchromatographie }}$ auf Aluminiumoxid $\mathrm{GF}_{2}$, System Benzol/Methylenchlorid/Essigsäureäthylester $(74: 32: 16 \mathrm{v} / \mathrm{v})$ nach dreimaliger Entwicklung

\begin{tabular}{ll}
\multicolumn{1}{c}{ Steroid } & $R_{\mathbf{F}}$ \\
\hline Epitestosteron & 0,41 \\
Ätiocholanolon & 0,49 \\
$17 \alpha$-Hydroxyprogesteron & 0,49 \\
Testosteron & 0,57 \\
Dehydroepiandrosteron & 0,66 \\
Androsteron & 0,70 \\
\hline
\end{tabular}

Ätiocholanolon auch sicher $\mathrm{zu}$ erfassen, wurde eine $2 \mathrm{~cm}$ breite Zone der Dünnschichtplatte ausgekratzt. Dabei wäre es möglich, daß auch ein Teil des Testosterons mit eluiert wird. Da aber Testosteron-dansylhydrazon und Ätiocholanolon-dansylhydrazon bei der 2. Dünnschichtchromatographie auf Kieselgel $\mathrm{H}$ in allen erprobten Lösungsmittelsystemen den gleichen

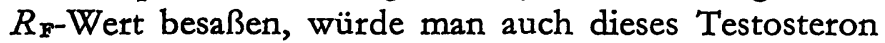
mitbestimmen. Wir fanden aber, daß sich die acetylierten Dansylhydrazone von Ätiocholanolon und Testosteron bei der Dünnschichtchromatographie auf Kieselgel $\mathrm{H}$ trennen ließen (Tab. 2). Aus diesem Grunde

Tab. 2

$R_{\text {F-Werte }}$ von acetylierten Oxosteroid-dansylhydrazonen, Dünnschichtchromatographie auf Kieselgel H, System Benzol/Essigsäureäthylester $(90: 10 \mathrm{v} / \mathrm{v})$ nach zweimaliger Entwicklung

\begin{tabular}{ll}
\multicolumn{1}{c}{ Steroid } & $R_{\text {F }}$ \\
\hline $17 \alpha$-Hydroxyprogesteron & 0,08 \\
Testosteron & 0,41 \\
Atiocholanolon & 0,50 \\
\hline
\end{tabular}

wurde der Verdampfungsrückstand des Eluates der 1. Dünnschichtplatte vor der Umsetzung mit Dansylhydrazin acetyliert. Durch diesen zusätzlichen Arbeitsschritt ließ sich die Spezifität der Methode verbessern. Für die Spezifität der Methode spricht ferner die recht gute Übereinstimmung der von uns ermittelten Werte mit jenen, die mit einer Doppelisotopenverdünnungsmethode (3) und mit einer gaschromatographischen Methode (4) bestimmt wurden.

\section{Richtigkeit}

Bei unseren ersten Wiederfindungsversuchen eluierten wir das Aluminiumoxid der 1. Dünnschichtplatte mit Methanol. Der Verdampfungsrückstand des Eluates wurde acetyliert und dann mit Dansylhydrazin umgesetzt. Nach der 2. Dünnschichtchromatographie auf 
Kieselgel $\mathrm{H}$ stellten wir fest, daß das Acetyl-ätiocholanolon-dansylhydrazon nicht auf die 2. Platte gelangt war, da es an beim Eluieren mit Methanol ins Eluat gelangtes Aluminiumoxid adsorbiert wird. Daraufhin führten wir die Elution mit Chloroform durch. Um zu verhindern, daß auch die mit Chloroform ins Eluat gelangenden geringen Mengen Aluminiumoxid das gebildete Acetyl-ätiocholanolondansylhydrazon adsorbieren, versetzten wir den Verdampfungsrückstand des Eluates vor der Umsetzung mit Dansylhydrazin mit Progesteron-bis-(dansylhydrazon), das an etwa vorhandenes Aluminiumoxid absorbiert wird. Das anschließend gebildete Acetyl-ätiocholanolon-dansylhydrazon wurde dann nicht mehr an das Aluminiumoxid gebunden. Das Progesteron-bis-(dansylhydrazon) stört die Bestimmung nicht, da diese Verbindung bei der 2. Dünnschichtchromatographie auf Kieselgel $\mathrm{H}$ bedeutend langsamer läuft als Acetyl-ätiocholanolondansylhydrazon. Tabelle 3 zeigt die Ergebnisse einer

Tab. 3

Ergebnisse von Wiederfindungsversuchen ohne und mit Zusatz von Progesteron-bis-(dansylhydrazon). $10 \mathrm{ml}$ Sammelplasma wurde je-
weils $0,1 \mu \mathrm{g}$ Ätiocholanolon zugesetzt

\begin{tabular}{cc}
\hline $\begin{array}{c}\text { Ohne Prodergefundenes Ätocholanolon }(\mu \mathrm{g}) \\
\text { (dansylhydrazon) }\end{array}$ & $\begin{array}{c}\text { Mit Progesteron-bis- } \\
\text { (dansylhydrazon) }\end{array}$ \\
\hline 0,033 & 0,056 \\
0,036 & 0,068 \\
0,038 & 0,079 \\
0,050 & 0,086 \\
0,053 & 0,086 \\
0,057 & 0,086 \\
0,061 & 0,088 \\
0,062 & $\overline{\mathbf{x}}=0,078$ \\
\hline$\overline{\mathbf{x}}=0,049$ & \\
\hline
\end{tabular}

Reihe von Wiederfindungsversuchen ohne und mit Zusatz von Progesteron-bis-(dansylhydrazon). Ohne Zusatz der Progesteronverbindung ist die Streuung der Werte größer und die Wiederfindung beträgt nur $49 \%$. Bei den mit Zusatz der Progesteronverbindung durchgeführten Wiederfindungsversuchen wurde dagegen eine Wiederfindung von $78 \%$ erzielt.

\section{Präzision}

Der Gehalt von Sammelplasma an freiem Ätiocholanolon wurde achtmal bestimmt. Als Mittel wurde eine Konzentration von $0,35 \mu \mathrm{g} / 100 \mathrm{~m} l$ gefunden. Die Standardabweichung betrug $\mathrm{s}=0,04 \mu \mathrm{g} / 100 \mathrm{~m} /$, der Variationskoeffizient $\mathrm{V}=11,4 \%$.

\section{Nachiveisgrenze}

Die Nachweisgrenze wurde nach KAISER (9) aus der 3s-Grenze der Streuung der Leerwerte berechnet; sie lag bei $0,006 \mu \mathrm{g}$ in einer Probe.

\section{Ätiocholanolonkonzentration des Plasmas gesunder Personen} Der Gehalt des Plasmas an freiem Ätiocholanolon wurde mit der angegebenen Methode bei 5 weiblichen und 12 männlichen gesunden Versuchspersonen bestimmt (Tab. 4). Die Werte lagen im Bereich von 0,09 bis $0,41 \mu \mathrm{g} / 100 \mathrm{~m} /$ mit einem Mittelwert von $0,25 \mu \mathrm{g} / 100 \mathrm{ml}$.
Tab. 4

Freies Ätiocholanolon im Plasma von Normalpersonen

\begin{tabular}{|c|c|c|}
\hline Alter (Jahre) & Geschlecht & $\begin{array}{c}\text { freies } \\
\text { Átiocholanolon } \\
(\mu \mathrm{g} / 100 \mathrm{ml})\end{array}$ \\
\hline 17 & q & 0,15 \\
\hline 30 & q & 0,29 \\
\hline 32 & q & 0,28 \\
\hline 32 & $\stackrel{+}{q}$ & 0,20 \\
\hline 55 & $\phi_{+}^{+}$ & 0,16 \\
\hline 17 & $c^{\circ}$ & 0,11 \\
\hline 18 & $0^{\prime \prime}$ & 0,27 \\
\hline 24 & $0^{\prime \prime}$ & 0,33 \\
\hline 24 & $0^{\circ}$ & 0,12 \\
\hline 27 & $\sigma$ & 0,16 \\
\hline 27 & $0^{\prime \prime}$ & 0,41 \\
\hline 29 & $0^{\prime}$ & 0,09 \\
\hline 30 & $\sigma^{\circ}$ & 0,32 \\
\hline 30 & $0^{\circ}$ & 0,34 \\
\hline 31 & $\sigma^{\circ}$ & 0,38 \\
\hline 32 & $0^{\prime \prime}$ & 0,40 \\
\hline \multirow[t]{2}{*}{37} & $0^{\prime}$ & 0,30 \\
\hline & \multicolumn{2}{|r|}{$\begin{array}{r}\overline{\mathbf{x}}=0,25 \\
\mathrm{~s}= \pm 0,10\end{array}$} \\
\hline
\end{tabular}

\section{Diskussion}

Im Gegensatz zu den konjugierten 17-Oxosteroiden Androsteron, Dehydroepiandrosteron und Ätiocholanolon kommt das freie, nicht konjugierte Ätiocholanolon in einer viel geringeren Konzentration im Plasma vor. Zur Diagnose des sog. Ätiocholanolonfiebers ist aber gerade die quantitative Bestimmung des freien Ätiocholanolons von Wichtigkeit. Wegen der geringen Konzentration dieses Steroids im Plasma sollte eine Bestimmungsmethode besonders empfindlich sein. Bei der Reaktion von Oxosteroiden mit Dansylhydrazin entstehen die entsprechenden Dansylhydrazone, die man durch Dünnschichtchromatographie teinigen kann und die sich infolge ihrer starken Fluoreszenz besonders gut zur Bestimmung kleinster Mengen von Oxosteroiden eignen. Die Empfindlichkeit der fluorometrischen Bestimmung ist mehr als $30 \mathrm{mal}$ so groß wie bei photometrischen Methoden (7). Bei unserer Methode geschieht die Extraktion des Plasmas und die Entfernung der Lipoide in der allgemein üblichen Weise. Durch eine 1. Dünnschichtchromatographie auf Aluminium- oxid wird eine Vorreinigung des Ätiocholanolons erreicht. Der Verdampfungsrückstand des Eluates wird acetyliert und dann mit Dansylhydrazin umgesetzt. Das gebildete Acetylätiocholanolon-dansylhydrazon trennt man durch eine 2. Dünnschichtchromatographie auf Kieselgel $a b$ und mißt seine Fluoreszenz nach dem Eluieren des Kieselgels. Die fluorometrische Messung erfolgt in Tetrachloräthan anstelle von Chloroform, da dieses Lösungsmittel infolge seines hohen Siedepunktes $\left(146^{\circ}\right)$ kaum verdunstet und das Volumen der Meßlösung konstant bleibt. Die von uns bei 17 Personen ermittelten Werte liegen im Bereich von 0,09 bis $0,41 \mu \mathrm{g} /$ $100 \mathrm{~m} l$. Gandy und Petrerson (3) fanden mit einer Doppelisotopenverdünnungsmethode Normalwerte $z$ wischen 0,01 und $0,27 \mu \mathrm{g} / 100 \mathrm{~m} l$ und die Normalwerte von KLORKE und ThIJSSEN (4), bestimmt mit einer gaschromatographischen Methode, lagen zwischen 0,08 und $0,17 \mu \mathrm{g} / 100 \mathrm{ml}$. Mit der 2,4-Dinitrophenylhydra- 
zin-Methode $(6,7)$ wurden jedoch höhere Normalwerte gefunden, die zwischen 0,5 und $1,2 \mu \mathrm{g} / 100 \mathrm{~m} l$ lagen.

Der Vorteil der von uns beschriebenen Methode liegt darin, daß sie sich ohne Gaschromatograph und auch ohne Verwendung radioaktiv markierter Verbindungen ausführen läßt. Die Nachweisgrenze liegt bei $0,006 \mu \mathrm{g}$, so daß sich die Bestimmung auch in $5 \mathrm{~m} / \mathrm{Plasma}$ durchführen lassen müßte. Wir haben zunächst mit $10 \mathrm{ml}$ Plasma gearbeitet, um bei der Bestimmung des freien Ätiocholanolons im Plasma gesunder Versuchspersonen auch die kleinsten Konzentrationen sicher erfassen zu können.

\section{Literatur}

1. Segaloff, A., C. Bowers, D. Gordon, J. Schlosser und P. Murrson, Cancer (Philadelphia) 10, 1116 (1957). - 2. Kappas, A., L. Hellman, D. Fukushima und T. Galiagher, J. Clin. Endocr. (Springfield) 17, 451 (1957). - 3. GANDY, H. M. und R. E. Peterson, J. Clin. Endocr. (Springfield) 28, 949 (1968). 4. KIOKKE, A. H. und J. H. H. ThrJssen, Clin. chim. Acta (Amster- dam) 20, 167 (1968). - 5. FekÉr, T. und L. HaLMY, Europ. J. Steroids 2, 443 (1967). - 6. CoHN, G. L., P. K. BoNDY und C. CAstigurone, J. Clin. Invest. 40, 400 (1961). - 7. Treiber, L. W. Rindt und G. W. Oerter, diese Z. 5, 102 (1967). - 8. Graep, V., diese Z. 8, 320 (1970). - 9. KAISER, H., Z. analyt. Chem. 209, 1 (1965).
Dr. Volkmar Graef 6300 Gießen

Friedrichstr. 24 